(C) Revista de Matemática: Teoría y Aplicaciones 2020 27(2) : 355-365

CIMPA - UCR ISSN: 1409-2433 (PRINT), 2215-3373 (ONLINE)

DOI: https://doi.org/10.15517/rmta.v27i2.34109

\title{
A STOCHASTIC MODEL FOR GERMINATION
}

\section{UN MODELO ESTOCÁSTICO DE GERMINACIÓN}

\author{
JosÉ VILLA-MORALES*
}

Received: 19/Oct/2018; Revised: 29/Nov/2019;

Accepted: 6/Feb/2020

Revista de Matemática: Teoría y Aplicaciones is licensed under a Creative Commons Reconocimiento-NoComercial-Compartirigual 4.0 International License.

Creado a partir de la obra en http://www.revistas.ucr.ac.cr/index.php/matematica

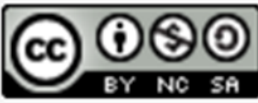

*Universidad Autónoma de Aguascalientes, Departamento de Matemáticas y Física, Aguascalientes, Mexico. E-Mail: jvilla@ correo.uaa.mx 


\begin{abstract}
Assuming that the germination process of a seed passes through several stages (or states), including a state of non-germination, we model this phenomenon by means of a continuous-time Markov chain. The distribution of the germination time and the average of the first germination is obtained. In particular, when the duration of the process at each stage is on average the same we see that the proposed model adjusts rather well some experimental data.
\end{abstract}

Keywords: seed germination; first germination time; continuous-time Markov chains.

\title{
Resumen
}

Asumiendo que el proceso de germinación de una semilla pasa por varias etapas (o estados), incluyendo un estado de no germinación, se modela éste fenómeno por medio de una cadena de Markov a tiempo continuo. Se obtienen la distribución del tiempo de germinación y la media del tiempo de la primera germinación. En particular, cuando la duración del proceso de germinación es en promedio el mismo en cada etapa vemos que el modelo propuesto se ajusta bastante bien a datos experimentales.

Palabras clave: germinación de semillas; tiempo de la primera germinación; cadenas de Markov a tiempo continuo.

Mathematics Subject Classification: 92B05, 60J05 60J27, 34A05.

\section{Introduction}

The development of plants has several stages, in this work we will study the first one, namely the germination stage. We are going to focus on the time of germination. More concretely, we will model the percentage of germination. Such model can be applied to predict germination rates and such information can be used to take different decisions, for example in the distribution of transplant time of the seedlings.

There are different ways to model the germination time (see $[2,4,5,6]$ and the references therein). Some of them are based on predictive equations and they are reasonably accurate over a defined range [3]. On the other hand, the cumulative percentage of germination time of experimental data suggests an Sshape germination curve and there are many attempts to fit such curve to the data [10]. Among the classic models, with S-shape, are those that use the Logistic [9, 13] or Richards function [1]. Since the data are stochastic, different experiment yields different points, this is why we believe that a stochastic approach is more 
helpful than a deterministic one. Based on this, in this paper, we propose a stochastic model for seed germination.

Germination is a biological process that is affected by various environmental as well as genetic factors. We will assume that such a complex biological process will be carried out in several stages beginning with water uptake by the seed and culminating in the emergence of the embryo from the seed coat (radical or hypocotyl emergence), see [6]. Our contribution here is that we will use the theory of pure jump Markov chains to model the germination process. Each stage of the germination process will be a state of the stochastic process, the state when there is no germination will be called cemetery state. We will assume that the time the seed lasts in one state has an exponential distribution and each stage is independent of each other. So we have a stochastic model in which we can determine the average time of the first germination, the time at which the change in the germination rate occurs (this is why we have an S-shape curve) and the accumulated germination percentage fit the data [2] quite well. Assuming the rate is constant between successive stages Thornley [12] found that the waiting time of germination has gamma distribution, we get this as particular case. Some very interesting models that also considers stages in the germination process appears in $[6,14]$, in these models a cemetery state is not considered as we do here.

The paper is organized as follows. In Section 2 we present the stochastic model and in Section 3 we discuss some important particular cases. In the last section we present some conclusions.

\section{Description of the stochastic model}

Let us consider a model for seed germination where $c, 1,2, \ldots, g-1, g$ represent the different states in the process of germination. Here $c$ is the cemetery state (the non germination of the seed) and $g$ is the germination state. Such states are absorbing states, this means that once the seed takes one of these two states the seed remains permanently in such state, the other states are non absorbing.

The germination model of a seed is as follows. At time 0 the seed is in state 1 (the water uptake by the seed). We suppose the seed remains there until some positive time $\tau_{1}$, after the seed jumps to one of the states $c$ or 2 with probability $a_{1}$ and $b_{1}$, respectively (then, $a_{1}+b_{1}=1$ ). If the seed reaches the state $c$ it remains there permanently. If the seed reaches the state 2 it remains there until some positive time $\tau_{2}$, at which time the seed jumps to one of the states $c$ or 3 with probability $a_{2}$ and $b_{2}$, respectively $\left(a_{2}+b_{2}=1\right)$. Again, if the seed reaches the state $c$ it remains there permanently, if not the process evolves as before. 
When the seed is in state $g-1$ it remains there until some positive time $\tau_{g-1}$, after the seed jumps to one of the states $c$ or $g$ with probability $a_{g-1}$ and $b_{g-1}$, respectively $\left(a_{g-1}+b_{g-1}=1\right)$, and remains there permanently. We will assume the times $\tau_{i}$ are exponentially distributed with density:

$$
f_{\tau_{i}}(t)=\lambda_{i} e^{-\lambda_{i} t}, \quad t \geq 0
$$

Under these considerations the stochastic process:

$$
X(t)=\left\{\begin{array}{cc}
x_{1}, & 0 \leq t<\tau_{1}, \\
x_{2}, & \tau_{1} \leq t<\tau_{2}, \\
x_{3}, & \tau_{2} \leq t<\tau_{3}, \\
\vdots & \vdots
\end{array}\right.
$$

is a Markov pure jump process, where $x_{1}=1, x_{i} \in\{c, i\}$ with $i \in\{2,3, \ldots$, $g-1, g\}$. The random variable $X(t)$ represent the state of the seed at time $t$.

Let us suppose the Markov pure jump process $X$, with state space $S=\{c, 1,2, \ldots, g-1, g\}$, is defined on a probability space $(\Omega, \mathcal{F}, P)$. For such process the corresponding $Q$-matrix is (see [8]):

$$
\left(q_{i, j}\right)=\left(\begin{array}{ccccccc}
0 & 0 & 0 & 0 & 0 & 0 & 0 \\
\lambda_{1} a_{1} & -\lambda_{1} & \lambda_{1} b_{1} & 0 & 0 & 0 & 0 \\
\lambda_{2} a_{2} & 0 & -\lambda_{2} & \lambda_{2} b_{2} & 0 & 0 & 0 \\
\lambda_{3} a_{3} & 0 & 0 & -\lambda_{3} & \lambda_{3} b_{3} & 0 & 0 \\
\vdots & \vdots & \vdots & \vdots & \vdots & \ddots & \vdots \\
\lambda_{g-1} a_{g-1} & 0 & 0 & 0 & 0 & -\lambda_{g-1} & \lambda_{g-1} b_{g-1} \\
0 & 0 & 0 & 0 & 0 & 0 & 0
\end{array}\right) .
$$

In this case the numbers $1 / \lambda_{i}>0$ represent the mean time the seed is in the state $i$. Moreover, $\lambda_{i} a_{i}$ and $\lambda_{i} b_{i}$ represent the rate of going from the state $i$ to the state $c$ or $i+1$, respectively (see [11]). If $P_{i, j}(t)$ denotes the probability that the process $X$ starting in state $i$ will be in state $j$ at time $t$, then we have the following system of ordinary differential equations, see [11] or [8].

$$
P_{i, j}^{\prime}(t)=\sum_{k \in S} P_{i, k}(t) q_{k, j}, t>0 .
$$

With initial condition :

$$
P_{i, k}(0)= \begin{cases}1, & i=k \\ 0, & i \neq k\end{cases}
$$


The resulting differential equations are, for $i \in\{c, 1, \ldots, g\}$ :

$$
\begin{aligned}
& P_{i, 1}^{\prime}(t)=-P_{i, 1}(t) \lambda_{1} \\
& P_{i, j}^{\prime}(t)=P_{i, j-1}(t) \lambda_{j-1} b_{j-1}-P_{1, j}(t) \lambda_{j} .
\end{aligned}
$$

for $j \in\{2,3, \ldots, g-1\}$ and:

$$
\begin{aligned}
P_{i, g}^{\prime}(t) & =P_{i, g-1}(t) \lambda_{g-1} b_{g-1} \\
P_{i, c}^{\prime}(t) & =P_{i, 1}(t) \lambda_{1} a_{1}+P_{i, 2}(t) \lambda_{2} a_{2}+\cdots+P_{i, g-1}(t) \lambda_{g-1} a_{g-1} .
\end{aligned}
$$

The recursive solution for such system, with initial condition (1), is:

$$
\begin{aligned}
P_{i, 1}(t) & =e^{-\lambda_{1} t} \\
P_{i, j}(t) & =\lambda_{j-1} b_{j-1} e^{-\lambda_{j} t} \int_{0}^{t} e^{\lambda_{j} r} P_{i, j-1}(r) d r \\
P_{i, g}(t) & =\lambda_{g-1} b_{g-1} \int_{0}^{t} P_{i, g-1}(r) d r \\
P_{i, c}(t) & =\int_{0}^{t}\left(\lambda_{1} a_{1} P_{i, 1}(r)+\lambda_{2} a_{2} P_{i, 2}(r)+\cdots+\lambda_{g-1} a_{g-1} P_{i, g-1}(r)\right) d r .
\end{aligned}
$$

The probability $P_{i, c}(t)$ can also be written as:

$$
P_{i, c}(t)=1-\sum_{j=1}^{g} P_{i, j}(t) .
$$

On the other hand, let us call $G$ the event of germination of a seed. The event $G$ occurs if and only if $\left\{X\left(\tau_{1}\right)=2, X\left(\tau_{2}\right)=3, \ldots, X\left(\tau_{g-1}\right)=g\right\}$, therefore there is germination with probability $b:=\prod_{k=1}^{g-1} b_{k}$.

Now suppose we sow $N$ seeds and they behave independently during the germination process. Given a time $t$ each of the $N$ seeds can be in one of the states $x \in S=\{c, 1,2, \ldots, g-1, g\}$ with probability $P_{1, x}(t)$. If $Y_{x}(t)$ is the number of seeds in state $x$ then $Y(t)=\left(Y_{c}(t), Y_{1}(t), \ldots, Y_{g}(t)\right)$ have multinomial distribution with parameter $N$ and $\left(P_{1, c}(t), P_{1,1}(t), \ldots, P_{1, g}(t)\right)$ and even more the marginal variables $Y_{x}(t)$ have binomial distribution with parameters $N, P_{1, x}(t)$. From this, the number of seeds germinated at time $t$ has expectation:

$$
E\left[Y_{g}(t)\right]=N P_{1, g}(t)
$$

and variance:

$$
\operatorname{Var}\left[Y_{g}(t)\right]=N P_{1, g}(t)\left(1-P_{1, g}(t)\right)
$$




\section{Discussion}

Now let us discuss some particular cases usually considered in the literature.

\subsection{There are three states in the germination process}

Let us assume that $S=\{c, 1,2\}$, here 2 is the germination state. In this case:

$$
\begin{aligned}
& P_{1,1}(t)=e^{-\lambda_{1} t}, \\
& P_{1,2}(t)=b_{1}\left(1-e^{-\lambda_{1} t}\right), \\
& P_{1, c}(t)=a_{1}\left(1-e^{-\lambda_{1} t}\right) .
\end{aligned}
$$

The germination time of one seed is $\tau_{1}$. If there are $N$ seeds the time for the first germination is $T_{1}:=\min \left\{\tau_{1}^{1}, \ldots, \tau_{1}^{N}\right\}$ where $\tau_{1}^{i}$ is the time the $i$-th seed takes to germinate. The time of the first germination $T_{1}$ has exponential distribution with parameter $N \lambda_{1}$, therefore $T_{1}$ has mean:

$$
E\left[T_{1}\right]=\frac{1}{N \lambda_{1}}
$$

and variance :

$$
\operatorname{Var}\left[T_{1}\right]=\frac{1}{N^{2}\left(\lambda_{1}\right)^{2}}
$$

Moreover :

$$
\begin{aligned}
E\left[Y_{2}(t)\right] & =N b_{1}\left(1-e^{-\lambda_{1} t}\right), \\
\operatorname{Var}\left[Y_{2}(t)\right] & =N b_{1}\left(1-e^{-\lambda_{1} t}\right)\left(1-b_{1}\left(1-e^{-\lambda_{1} t}\right)\right) .
\end{aligned}
$$

The mean of germinable seeds is $N-E\left[Y_{2}(t)\right]-E\left[Y_{c}(t)\right]$, then:

$$
N-E\left[Y_{2}(t)\right]-E\left[Y_{c}(t)\right]=N e^{-\lambda_{1} t} .
$$

This case corresponds to the linear relationship, found in [4], between the logarithm of the number of germinable seeds and the time.

\subsection{The average time in each stage of the germination process is constant}

Now let us consider the case when $\lambda_{i}=\lambda$, for $i=1,2, \ldots, g-1$, this means the average time in each state does not depend on this one. In this case we can have 
an explicit solution to the system (2):

$$
\begin{aligned}
P_{1,1}(t) & =e^{-\lambda t} \\
P_{1, j}(t) & =\left(\prod_{k=1}^{j-1} b_{k}\right) \frac{\lambda^{j-1}}{(j-1) !} t^{j-1} e^{-\lambda t} \\
P_{1, g}(t) & =\left(\prod_{k=1}^{g-1} b_{k}\right) \frac{\lambda^{g-1}}{(g-2) !} \int_{0}^{t} e^{-\lambda r} r^{g-2} d r
\end{aligned}
$$

Otherwise, conditioning on germination, the germination time $T_{g}$ is given by $\tau_{1}+\tau_{2}+\cdots+\tau_{g-1}$, where each random variable $\tau_{i}$ has exponential distribution with parameter $\lambda$, therefore the conditional distribution of $T_{g}$ is a gamma distribution with parameters $g$ and $g \lambda$, that is the conditional density function is given by:

$$
f_{T_{g}}(t)=\frac{\lambda^{g-1} t^{g-2}}{(g-2) !} e^{-\lambda t}, \quad t \geq 0
$$

One easy way to deduce (3) is observing that:

$$
\{X(t)=g\}=\left\{T_{g} \leq t\right\} \cap G,
$$

then:

$$
P_{1, g}(t)=P(X(t)=g \mid X(0)=1)=P(G) P\left(T_{g} \leq t \mid X(0)=1, G\right) .
$$

Remember that $P(G)=\prod_{k=1}^{g-1} b_{k}$.

Using mathematical induction (on $\mathrm{g}$ ) it can be proved that:

$$
\int_{0}^{t} e^{-\lambda r} r^{g-2} d r=\frac{(g-2) !}{\lambda^{g-1}}-\frac{e^{-\lambda t}}{\lambda}\left(t^{g-2}+\frac{(g-2) t^{g-3}}{\lambda}+\cdots+\frac{(g-2) !}{\lambda^{g-2}}\right),
$$

then (3) implies:

$$
P_{1, g}(t)=\left(\prod_{k=1}^{g-1} b_{k}\right)\left[1-e^{-\lambda t} \sum_{k=0}^{g-2} \frac{(\lambda t)^{k}}{k !}\right]
$$

Therefore, when the cemetery state is not considered in the model we get as particular case the model introduced in [14].

If we have $N$ seeds then:

$$
E\left[Y_{g}(t)\right]=N\left(\prod_{k=1}^{g-1} b_{k}\right)\left[1-e^{-\lambda t} \sum_{k=0}^{g-2} \frac{(\lambda t)^{k}}{k !}\right] .
$$

Rev.Mate.Teor.Aplic. (ISSN print: 1409-2433; online: 2215-3373) Vol. 27(2): 355-365, Jul-Dec 2020 
Therefore, the percentage of seeds germinated at time $t$ is on average:

$$
\frac{E\left[Y_{g}(t)\right]}{N} \times 100
$$

As we will see this function on $t$ has an S-shape and can be used to fit the germination data given in [2].

If we denote by $\tilde{P}(\cdot)=P(\cdot \mid G)$ the conditional probability on germination, then the first time to germination have men:

$$
\begin{aligned}
\tilde{E}\left[\min \left\{T_{g}^{1}, \ldots, T_{g}^{N}\right\}\right] & =\int_{0}^{\infty} \tilde{P}\left(\min \left\{T_{g}^{1}, \ldots, T_{g}^{N}\right\}>t\right) d t \\
& =\int_{0}^{\infty}\left(\prod_{k=1}^{N} \tilde{P}\left(T_{g}^{k}>t\right)\right) d t \\
& =\int_{0}^{\infty}\left(\tilde{P}\left(T_{g}^{k}>t\right)\right)^{N} d t \\
& =\int_{0}^{\infty}\left(1-\tilde{P}\left(T_{g}^{k} \leq t\right)\right)^{N} d t \\
& =\int_{0}^{\infty} e^{-N \lambda t}\left(\sum_{k=0}^{g-2} \frac{(\lambda t)^{k}}{k !}\right)^{N} d t
\end{aligned}
$$

where $\tilde{E}$ is the expectation with respect to the conditional probability measure $\tilde{P}$.

\subsection{Fitting an experimental data}

In this subsection we are going to consider the experimental data of Bould and Abrol [2]. Let us suppose that 100 seeds of tomato are sown, under constant temperature, of which 98 germinated. In this case $N=100$ and we can take for the probability of germination $b=98 / 100=0.98$. As in [14] we will suppose the model consider 9 stages and in each of them the seed stays a mean time of $1 / \lambda=1.8532 /$ days. Under this considerations the function to fit the data is (see (4))

$$
M(t)=(100)(0.98)\left[1-e^{-(0.5396) t} \sum_{k=0}^{7} \frac{((0.5396) t)^{k}}{k !}\right], t \geq 0 .
$$

In Figure 1 we can see how well the function $M$ fits the data given in [2]. In particular, if we take $\frac{d^{2}}{d t^{2}} M(t)=0$ we see that the rate of germination grows below 


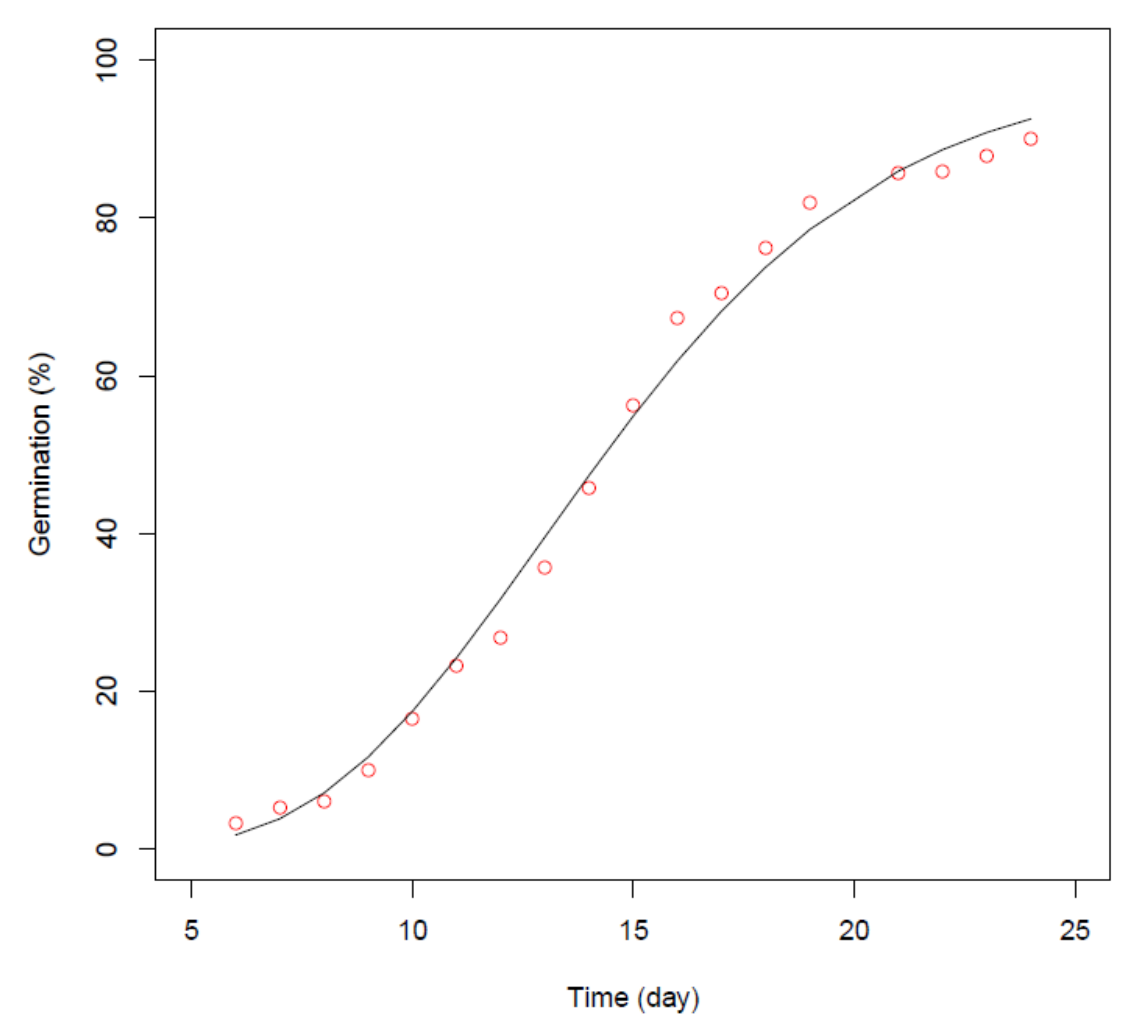

Figure 1: The continuous line is the function $M(t)$ and red $\circ$ are the experimental data in [2].

$t=12.973$ and decreases above $t=12.973$. This means that, the maximum rate of germination is at the 13 th day of sowing the seeds.

On the other hand, conditioning on the event that there is germination, the expected time for the first germination is:

$$
\int_{0}^{\infty} e^{-100(0.5396) t}\left(\sum_{k=0}^{7} \frac{((0.5396) t)^{k}}{k !}\right)^{100} d t=5.008
$$

which is very close with the day reported in [2] (see Figure 1). 


\section{Concluding remarks}

Using the tools of continuous-time Markov chains we get a unambiguous, amenable and a concise germination model. Moreover the proposed model is consistent with the existing literature about it and the data are very well fitted. The parameters in the model have a specific interpretation, for example, for the data in [2] we have the cemetery state, the germination state and seven intermediate stages, each of them with a duration of almost two days on average $(1 / 0.5396=1.8532)$. The stochastic model suggests that, in general, germination data do not follow a normal distribution, as many research assume [7]. It is more natural to assume that the data come from a gamma distribution. Indeed, from Figure 1 we see that the sample mean is between the 14th and 15th day and if we assume that the data in [2] come from a gamma distribution with parameters 8 and 0.5396 , then the mean is 14.8257 and moreover the median is 14.2128. We see how well the mean of a gamma distribution predicts the sample mean. It is worth mention that the variance is (27.4755) larger than desired and this may be due to intrinsic factors of the seeds themselves (weight of the seeds, for example).

\section{Acknowledgements}

Many thanks to the referees for their valuable and constructive observations that allowed a considerable improvement of the manuscript.

\section{Funding}

The author was partially supported through grant PIM20-1 of Universidad Autónoma de Aguascalientes.

\section{References}

[1] G.J. Barry, R.J. Cawood, R.G. Flood, Curve fitting of germination data using the Richards function, Plant, Cell and Environment, 11(1988), no. 3, 183-188. Doi: 10.1111/j.1365-3040.1988.tb01135.x

[2] A. Bould, B.K. Abrol, A model for seed germination curves, Seed Sci and Tech, 9(1981), no. 2, 601-611.

[3] H. Challa, Prediction of production: requisite of an integrated approach, Acta Hortic. 229(1988), 133-141. Doi: 10.17660/ActaHortic. 1988.229 .11 
[4] A.A. Goloff Jr., F.A. Bazzaz, A germination model for natural seed population, J. Theor. Biol. 52(1975), no. 2, 259-283. Doi: $10.1016 /$ $0022-5193(75) 90001-6$

[5] M.K. Gupta, P. Chandra, D.V.K. Samuel, B. Singh, A. Singh, M.K. Garg, Modeling of tomato seedling growth in greenhouse, Agric. Res. 1(2012), 362-369. Doi: $10.1007 / \mathrm{s} 40003-012-0035-5$

[6] Y. Hara, Estimating the temperature dependence of germination time by assuming multiple rate-determining steps, Plant. Prod. Sci. 8(2005), no. 4, 361-367. Doi: $10.1626 /$ pps. 8.361

[7] H.O. Harley, The modified Gauss-Newton method for the fitting of nonlinear regresion function by least squares, Technometric 3(1961), no. 2, 269-280. Doi: $10.2307 / 1266117$

[8] P.G. Hoel, S.C. Port, C.J. Stone, Introduction to Stochastic Processes, Houghton Mifflin, Boston, 1972.

[9] F.H. Hsu, C.J. Nelson, W.S. Chow, A mathematical model to utilize the logistic function in germination and seedling growth, J. Expl. Bot. 351(1984), no. 11, 1629-1640. doi: $10.1093 / \mathrm{jxb} / 35.11 .1629$

[10] J.G.M. Janssen, A method of recording germination curves, Ann. Bot. 37(1973), no. 4, 705-708. doi: 10.1093 /oxfordjournals.aob. a 084738

[11] J.R. Norris, Markov Chains, Cambridge University Press, 1997. doi: 10 . $1017 / \mathrm{CBO} 9780511810633$

[12] J.H.M. Thornley, Germination of seeds and spores, Annals of Botany 41(1977), no. 6, 1363-1365. doi: 10 . 1093 / oxfordjournals .aob. a 085425

[13] M. Torres, G. Frutos, Logistic function analysis of germination behaviour of age fennel seeds, Enviromental and Experimental Botany, 30(1990), no. 3, 383-390. doi: $10.1016 / 0098-8472$ (90) 90051-5

[14] S. Tseng, J.P. Hsu, A stochastic analysis of seed germination, Botanic Bulletin of the Academia Sinica 30(1989), 201-205.

Rev.Mate.Teor.Aplic. (ISSN print: 1409-2433; online: 2215-3373) Vol. 27(2): 355-365, Jul-Dec 2020 
